\title{
AN APPARATUS FOR THE DIRECT MEASUREMENT OF COLLIMATOR TRANSVERSE WAKEFIELDS *
}

\author{
$\underline{\text { P. Tenenbaum }}^{\dagger}$, K. Bane, J. Irwin, R.K. Jobe, D. McCormick, T.O. Raubenheimer, M.C. Ross, \\ G. Stupakov, D. Walz \\ Stanford Linear Accelerator Center, Stanford, CA
}

\begin{abstract}
The design of the NLC Beam Delivery System requires a firmer understanding of the effects of collimators on short, intense bunches than is presently available. We describe an experiment to directly measure these effects through use of a dedicated apparatus located at the $1.19 \mathrm{GeV}$ point in the SLAC Linac. The apparatus consists of an outer vacuum vessel and an interchangeable insertion containing up to 5 distinct collimator apertures. The insertion is capable of remote-controlled translation, allowing the collimator apertures to be misaligned relative to the electron beam without changing the incoming beam orbit; the wakefield deflection is then measured by observing the change in the outgoing orbit on 32 beam position monitors. The parameters of the apertures have been selected to allow confirmation of the scaling laws for collimator wakefields, and to strongly enhance either the geometric or resistive wall contribution of each aperture. Details of the apparatus design, the aperture parameters, and the experimental program are discussed.
\end{abstract}

\section{INTRODUCTION}

The Next Linear Collider (NLC) post-linac collimation section is a $2.5 \mathrm{~km}$ long section of beamline the purpose of which is to prevent particles at large amplitudes from entering the final focus, where they would cause unacceptable detector backgrounds. The design of the NLC collimation section is substantially based upon experience at the Stanford Linear Collider (SLC) with a similar collimation system interleaved with accelerator structures in the last 300 meters of the linac.

One of the central design specifications of the NLC collimation system is the contribution to luminosity degradation from collimator short-range dipole wakefields. A beam which passes off-center through a collimator experiences a deflection due to wakefields; therefore a collimation system will amplify incoming jitter. The NLC collimation system has been limited to a $25 \%$ increase in beam jitter due to collimator wakefields.

The present understanding of collimator wakefields is not adequate to confidently predict the jitter amplification of a given collimator design. The NLC collimators are expected to utilize a smooth taper to match between a rectangular aperture and a cylindrical beampipe. The analytic expression for the deflection of an electron bunch due to

\footnotetext{
${ }^{*}$ Work supported by the Department of Energy, contract DE-AC0376 SF00515.

† Email:quarkpt@slac.stanford.edu
}

the geometric wakefield of such a collimator is [1]:

$$
\Delta y^{\prime}=2 \frac{N r_{e}}{\gamma} \frac{\pi h}{2 \sqrt{\pi}} \frac{\left(r_{0}-r_{1}\right)\left(r_{0}^{2}-r_{1}^{2}\right)}{\sigma_{z} L_{T} r_{0}^{2} r_{1}^{2}} y_{0},
$$

where $y_{0}$ is the beam offset in the collimator, $N$ is the bunch population, $r_{e}$ is the classical electron radius, $\gamma$ is the relativistic factor, $\sigma_{z}$ is the RMS bunch length, $L_{T}$ is the taper length, $r_{0}$ and $r_{1}$ are the radius of the beampipe and half-aperture of the gap, respectively, and $h$ is the transverse width of the gap (e.g., the horizontal width of a vertical collimator). As expected, the deflection in Equation 1 vanishes when $r_{1} \rightarrow r_{0}$; however, the deflection is also proportional to $h$, implying that the deflection from an infinitely-wide collimator diverges.

The resistive contribution to the wakefield deflection is expected to be [2]:

$$
\Delta y^{\prime}=2 \frac{\alpha_{R}}{\pi^{3 / 2}} \frac{e^{2} N c L_{T}}{E \sqrt{\sigma_{z}}} \sqrt{\frac{Z_{0}}{\sigma}} \frac{r_{0}+r_{1}}{r_{0}^{2} r_{1}^{2}}\langle f\rangle y_{0},
$$

where $Z_{0}$ is the impedance of free space, $\sigma$ is the conductivity of the collimator, $e$ is the electron charge, $E$ is the beam energy, $\langle f\rangle$ is a constant arising from integration of the kick over the (presumed) Gaussian distribution in $z(\langle f\rangle \approx 0.724)$, and $\alpha_{R}$ is a form factor equal to 1 for a cylindrical collimator and equal to $\pi^{2} / 8$ for a rectangular collimator. Note that Equation 2 does not contain nonphysical divergences and predicts a deflection even from a perfectly regular vacuum pipe $\left(r_{0}=r_{1}\right)$. However, measurements of the deflection due to the wakefields from the SLC collimators have exceeded those predicted by Equations 1 and 2 by up to a factor of 4 [3].

Collimator wakefield measurements $[3,4,5]$ have historically been difficult to interpret because they have been made on existing collimator installations, which are designed to minimize wakefield contributions rather than to amplify certain features of the wakefields over others. For example, the SLC collimator study discussed in [3] attempted to separate the resistive and geometric wakes by measuring the deflection as a function of bunch length: since Equation 1 predicts that $\Delta y^{\prime} \propto 1 / \sigma_{z}$, while Equation 2 predicts that $\Delta y^{\prime} \propto 1 / \sqrt{\sigma_{z}}$, the contributions from the two components could be inferred by such a measurement. However interpretation of such a measurement is far from straightforward due to uncertainty in the actual bunch length, response of beam monitors in the SLC under conditions of changing bunch length, and the impact of bunch length on the wakefields from the RF structures which fill the linac and complicate any trajectory measurement. A 
more optimal measurement could be made at a dedicated facility, in which collimators of any design could be tested. As part of the NLC research project at SLAC, we have designed such a facility.

\section{OVERALL DESIGN}

The collimator wakefield apparatus is a vacuum vessel with approximate dimensions 1.7 meters $\times 0.6$ meters $\times 0.3$ meters, as shown in Figure 1. The vessel is designed with a lid which can easily be removed and replaced to allow access to the interior. The vessel contains an insertion, which is a long block of aluminum containing 5 apertures; each aperture can be fitted with a collimator of any geometry desired.
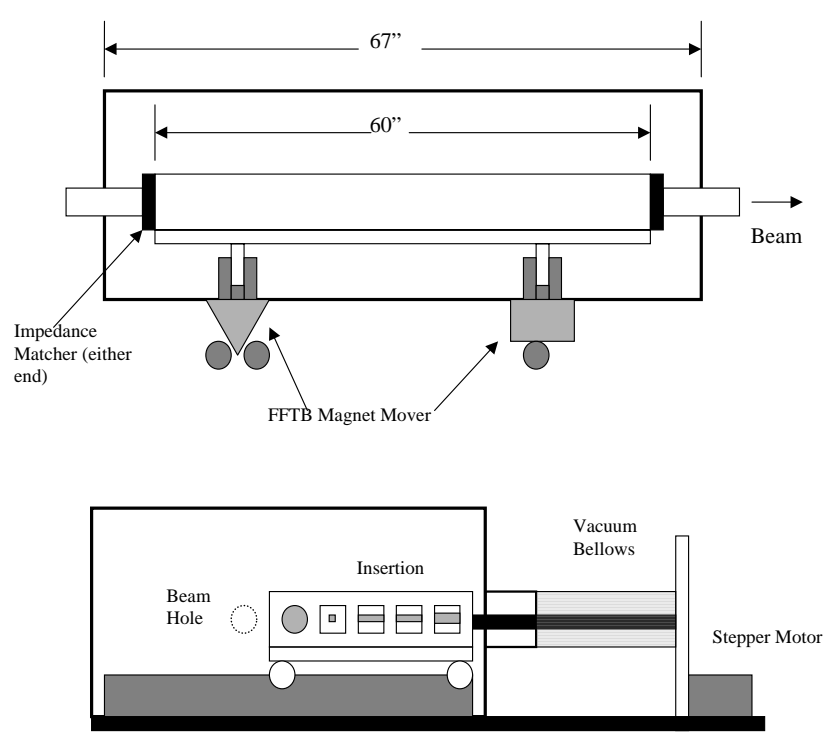

Figure 1: Diagram of collimator wakefield test apparatus, side view (top) and end view (bottom). The long, coarse translation stage and stepper motor move the insertion in $x$ to engage a test aperture; the FFTB magnet mover then moves the entire vacuum vessel in $y$ to produce a beamaperture displacement, which results in a measurable deflection.

The cartridge rides on a platform which is connected to an external horizontal translation stage; the stage engages whichever of the apertures is to be used for experimentation. In this way a series of related collimators can be tested in sequence without entering the accelerator housing or disturbing the vacuum vessel. When the translation stage moves to its full-out position, the insertion is removed completely from the path of the beam.

The vacuum vessel is mounted on a vertical translation stage which has a range of \pm 1.5 millimeters and a step size of $1 \mu \mathrm{m}$. Once one of the test apertures is engaged, the vertical stage is used to make a precise translation of the apparatus. The wakefields due to the collimator in the aperture will produce a deflection, which can be observed on beam position monitors downstream of the apparatus.
Wakefield deflections are inversely proportional to beam energy, and therefore the apparatus should be installed at a location where the beam energy is relatively low. The location selected is the SLAC linac spin rotator location immediately downstream of the point where the damped $1.19 \mathrm{GeV}$ beams re-enter the linac. This location is just upstream of the ASSET location, which allows a similar experiment on RF structures using a drive beam from the positron damping ring and a witness beam from the electron damping ring[6]. The collimator wakefield experiment will require only one beam. The deflection will be measured by recording the beam position on each of 32 beam position monitors downstream of the apparatus location, as well as several monitors upstream of the apparatus. The deflection will be reconstructed after pulse-to-pulse jitter is subtracted using the upstream BPMs. Because the downstream BPMs are largely interleaved with accelerator structures, it is desirable to use a low bunch charge so that the wakefields from the structures can be neglected. Our test apertures are designed to produce a measurable deflection for a bunch charge of $1 \times 10^{10}$. This is sufficiently below the threshold of instability in the linac that, if additional resolution is called for, the bunch charge can be increased moderately for some measurements.

\section{INSERTION DESIGN}

The bulk of the insertion should be constructed from a material with a high conductivity in order to avoid producing unwanted resistive-wall wakefields; it should be as light as possible to facilitate installation and removal in the cramped experimental area; and it should be made from a material which is easy to machine. On the basis of these criteria, aluminum was chosen for the material.

The insertion contains 1 circular beam channel and 4 square ones, each with a full bore of $4 \mathrm{~cm}$. The total length of the insertion is 1.5 meters, in order to accomodate the longest collimators foreseen for measurement. In order to simplify installing and removing collimator apertures from the channels, it was constructed in a split configuration with a top half and a bottom half which can be disassembled and reassembled easily.

The clearance between the insertion and the impedancematching flanges at either end of the vacuum vessel is 1 $\mathrm{mm}$. This produces a small discontinuity wakefield, but the clearance was deemed necessary to ensure that the insertion could move easily on its horizontal stage. The deflection resulting from the discontinuity is believed too small to measure, but this will be checked by engaging the cylindrical channel without any collimator aperture and measuring the deflection as a function of the vertical stage position. This will allow us to subtract the deflection due to the vacuum vessel, discontinuities, and other construction features. 


\section{APERTURE DESIGN}

In order to separately measure the contributions of geometric, resistive, surface finish, etc. wakefields, it is necessary to carefully construct each test aperture to enhance one particular source of deflections and reduce the rest. The first set of apertures are intended to maximize geometric wakefields. Therefore they are all constructed from copper, with a good finish, and all the apertures are short in $z$.

Table 1 shows the parameters of the first set of test apertures to be measured. Note that the predicted deflections are calculated for a round pipe tapering down to either a round or a rectangular aperture; we approximate these with a square pipe tapering down to a square aperture in the former case, and a square pipe tapering down to a rectangular aperture in the latter. Computation of the wakefields for our precise geometry is difficult and uncertain, but the expected deviation from the cylindrical approximation is on the order of $20 \%$. The expected resolution of the technique, based on ASSET measurements, is 1 microradian, and all of the expected deflections are large relative to this.

Table 1: Parameters and predicted deflections for geometric wakefield test apertures, given a beam charge of $1 \times 10^{10}$, an energy of $1.19 \mathrm{GeV}$, and a $1 \mathrm{~mm}$ apparatus vertical offset.

\begin{tabular}{|c|c|c|c|}
\hline $\begin{array}{c}\text { Channel } \\
\text { Number }\end{array}$ & $\begin{array}{c}\text { Aperture } \\
(\mathrm{cm})\end{array}$ & $\begin{array}{c}\text { Taper } \\
\text { Length }(\mathrm{cm})\end{array}$ & $\begin{array}{c}\text { Kick } \\
(\mu \mathrm{rad})\end{array}$ \\
\hline \hline 1 & 4.0, round & None & $\approx 0$ \\
\hline 2 & 0.4, square & 5.0 & 1.9 \\
\hline 3 & 0.4, rect. & 5.0 & 51.3 \\
\hline 4 & 0.4, rect. & 10.0 & 33.9 \\
\hline 5 & 0.8, rect. & 5.0 & 25.6 \\
\hline
\end{tabular}

The second set of test apertures will measure resistivewall wakefields. This requires as axisymmetric a geometry as possible, in order to minimize geometric-wake contributions. The resistive wakefield can be further enhanced by using a material with a poor conductivity and including a long untapered section with a small gap in between the two tapers, which should enhance resistive deflections but leave geometric deflections unaffected. Ideally these measurements would use a second insertion in which all channels are cylindrical; machining considerations may mandate use of a square aperture once again. Table 2 shows the desired parameters for a resistive-wall wakefield test insertion. While we have used stainless steel as our resistive material in Table 2, we are presently leaning towards use of a pure element for this purpose, in order to achieve a uniform conductivity which does not depend upon relative densities of various alloyed materials in the surface.

Future insertions will test the effects of a subset of geometric features, namely surface finish and surface features, and also probe the behavior when geometric collimators of varying width are used. Ultimately an NLC-design colli-
Table 2: Parameters and predicted deflections for resistive wakefield test apertures, given a beam charge of $1 \times 10^{10}$, an energy of $1.19 \mathrm{GeV}$, and a $1 \mathrm{~mm}$ apparatus vertical offset. Test apertures 1 and 2 are copper, while 3 through 5 are steel.

\begin{tabular}{|c|c|c|c|c|}
\hline $\begin{array}{c}\text { Coll. } \\
\#\end{array}$ & $\begin{array}{c}\text { Aperture } \\
(\mathrm{cm})\end{array}$ & $\begin{array}{c}\text { Taper } \\
\text { Length }(\mathrm{cm})\end{array}$ & $\begin{array}{c}\text { Untapered } \\
\text { Length }(\mathrm{cm})\end{array}$ & $\begin{array}{c}\text { Kick } \\
(\mu \mathrm{rad})\end{array}$ \\
\hline \hline 1 & 0.4 & 5.0 & 0 & 1.9 \\
\hline 2 & 0.4 & 5.0 & 100 & 2.6 \\
\hline 3 & 0.4 & 5.0 & 100 & 7.1 \\
\hline 4 & 0.4 & 5.0 & 50 & 4.5 \\
\hline 5 & 0.8 & 20.0 & 100 & 3.2 \\
\hline
\end{tabular}

mator will be directly tested in this apparatus.

\section{EXPERIMENTAL PROGRAM}

The vacuum vessel and insertion with the first set of test apertures is presently under construction, with installation in the SLAC linac scheduled for late April. Once the translation stages are fully commissioned, experimental measurements will be made parasitically during PEP-II collisions, as it is expected that the PEP-II program can be interleaved with collimator studies with minimal disruption of either program. Measurements are expected to continue on a periodic basis for the next several years.

\section{ACKNOWLEDGEMENTS}

The authors wish to thank G. Bowden and D. Burke for their ideas and encouragement, and B. Brugnoletti for designing the test apparatus.

\section{REFERENCES}

[1] G. Stupakov, "Geometrical Wake of a Smooth Flat Collimator,” SLAC-PUB-7167 (1996).

[2] NLC ZDR Design Group, "Zeroth-Order Design Report for the Next Linear Collider," SLAC Report 474, 589 (1996).

[3] F. Zimmermann et al, "Measurements of Wakefields Generated by the Newly Coated Collimators in the SLAC Linac," SLAC-CN-415 (1997).

[4] F.J. Decker et al, "Design and Wake Field Performance of the New SLC Collimators,” Proc. Linac 96, 137 (1996).

[5] K.L.F. Bane et al, "Measurement of the Effect of Collimator Generated Wake Fields on the Beams in the SLC," Proc. PAC 95, 3031 (1995).

[6] C. Adolphsen et al, "Measurement of Wake Field Suppression in a Detuned X Band Accelerator Structure," Phys Rev Lett 74:2475 (1995). 